\title{
Retinal adaptation to spatial correlations
}

Kristina Simmons*, Jason Prentice ${ }^{+}$, Jan Homann ${ }^{+}$, Gasper Tkacik ${ }^{\wedge}$, Philip Nelson ${ }^{+}$, Vijay Balasubramanian*+

Departments of ${ }^{*}$ Neuroscience and ${ }^{+}$Physics, University of Pennsylvania $\wedge$ IST Austria

\section{Introduction}

Retinal ganglion cell (RGC) receptive fields are traditionally described in terms of an excitatory center with a concentric inhibitory surround. More recent white noise experiments do not show strong surrounds (Chichilnisky \& Kalmar, 2002). This might be evidence for weakening of the surround in the absence of correlations, as predicted by efficient coding theory. We tested for such adaptation by measuring receptive fields using white and exponentially correlated noise.

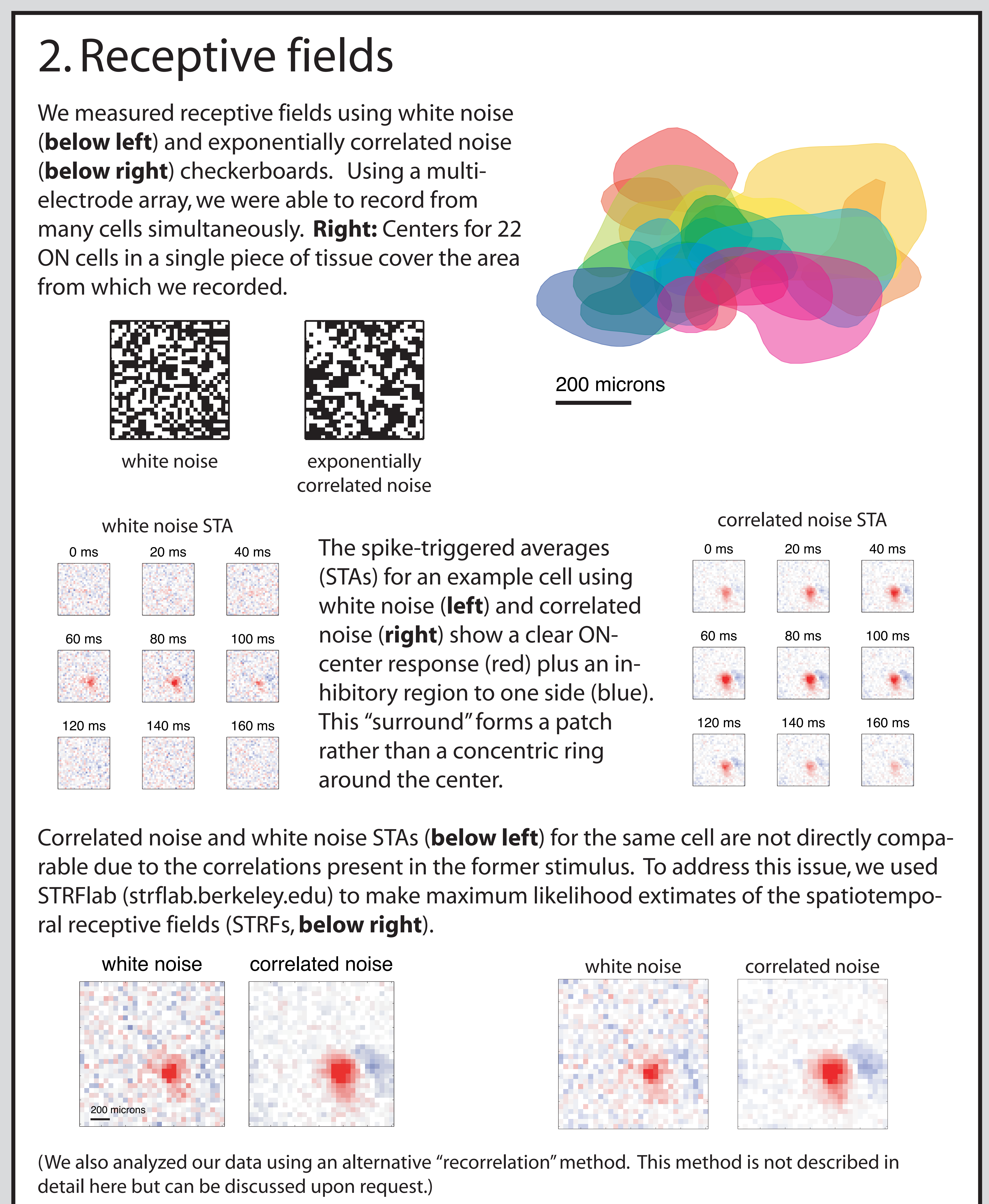

\section{Surround strength}

Our receptive fields are generally not well fit as a difference-of-gaussians model, so we developed a measure of relative surround strength that does not depend on fitting.

For each spatiotemporal receptive field, we first used principal components analysis to create a single spatial frame (below left). We then applied a threshold and a majority vote algorithm to find pixels that belonged to the center and surround regions (center). Next we took the sum or hix pixels in eachreglon at each timepoint to generate temporal receptive of the peak of the surround timecourse to the peak of the center timecourse.

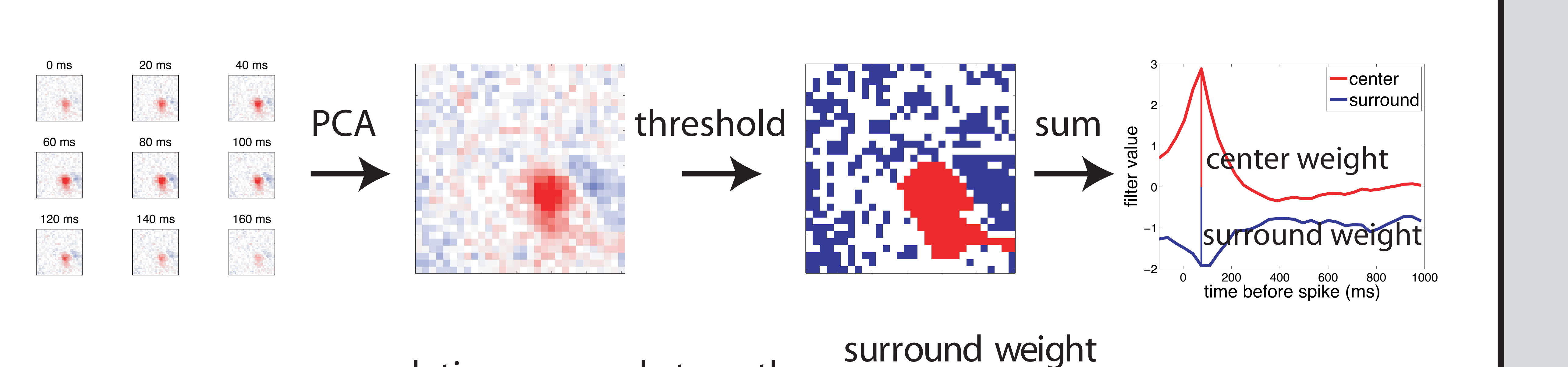

relative surround strength $=$ surround weight center weight

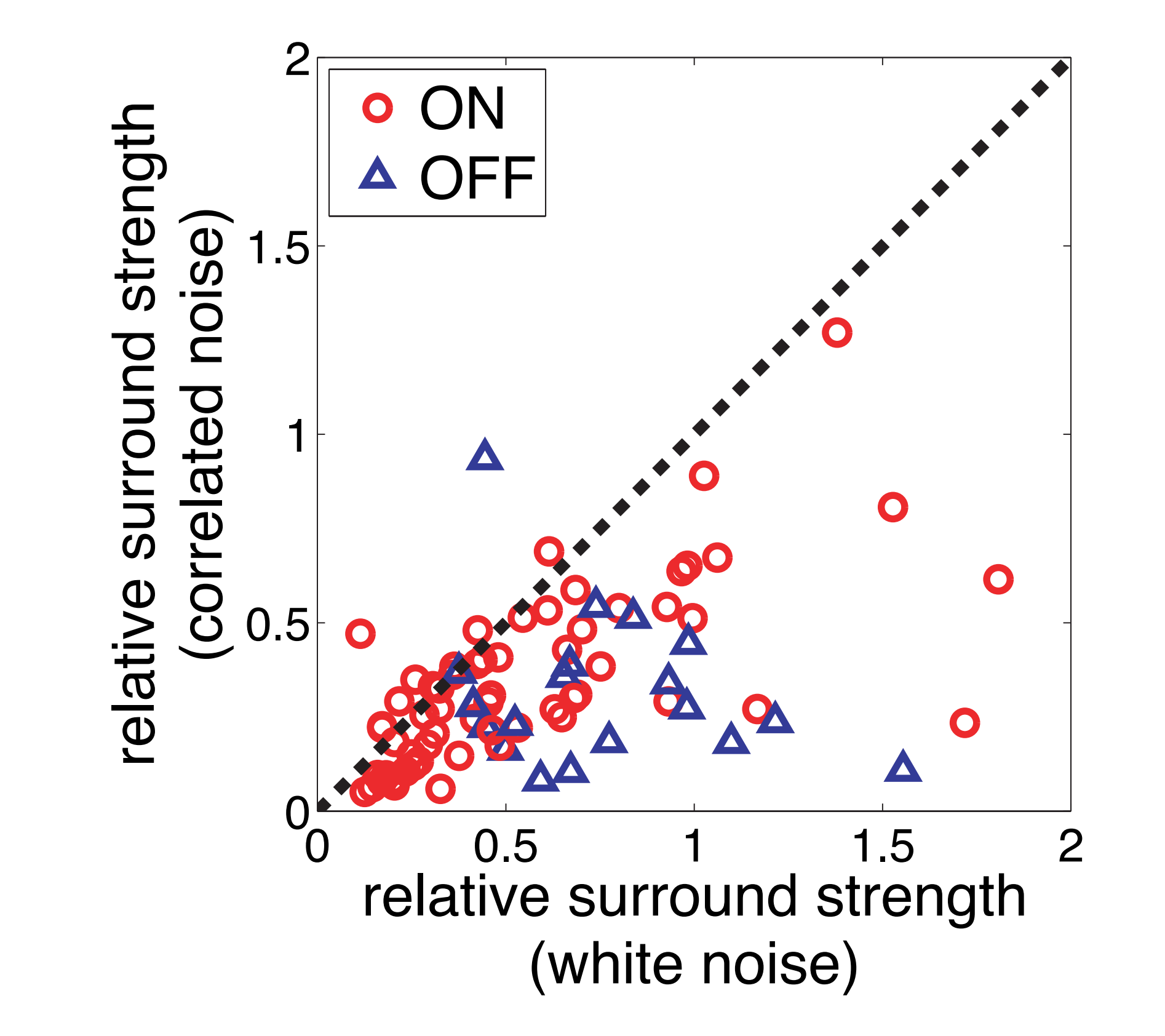

Left: Scatterplot of surround strengths shows that overall, cells tend to have stronger surrounds under white noise than under correlated noise. $\mathrm{N}=67 \mathrm{ON}$ cells, 26 OFF cells.

Right: We computed an adaptation index $\frac{\text { corr }- \text { white }}{\text { corr }+ \text { white }}$

for each cell. In both ON and OFF cells, most receptive fields have stronger relative surrounds
under white noise than under correlated noise.

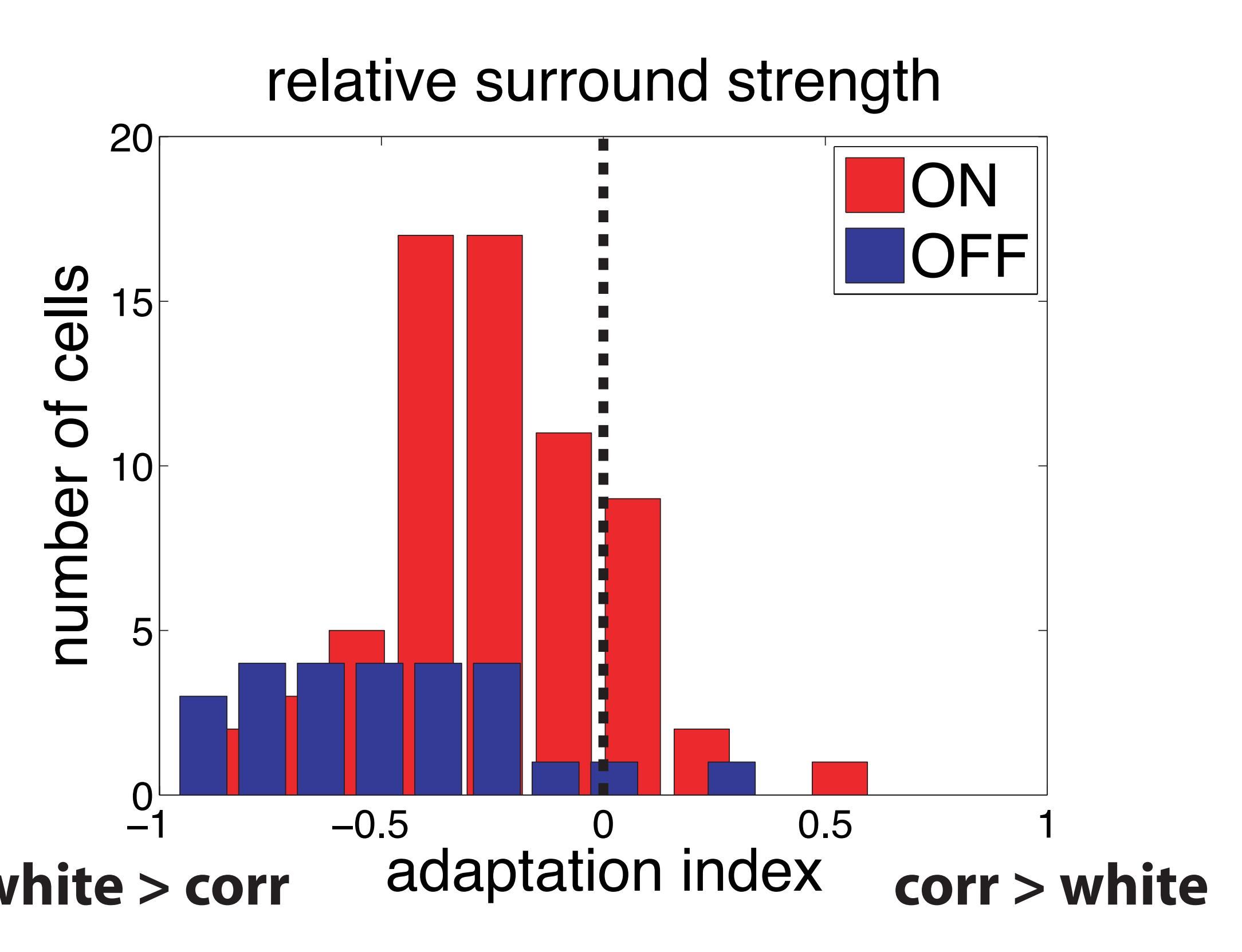

This difference in surround strength was produced by a larger center under correlated noise than under white noise
(near right) with no (near right) with no
change in surround size (far right).

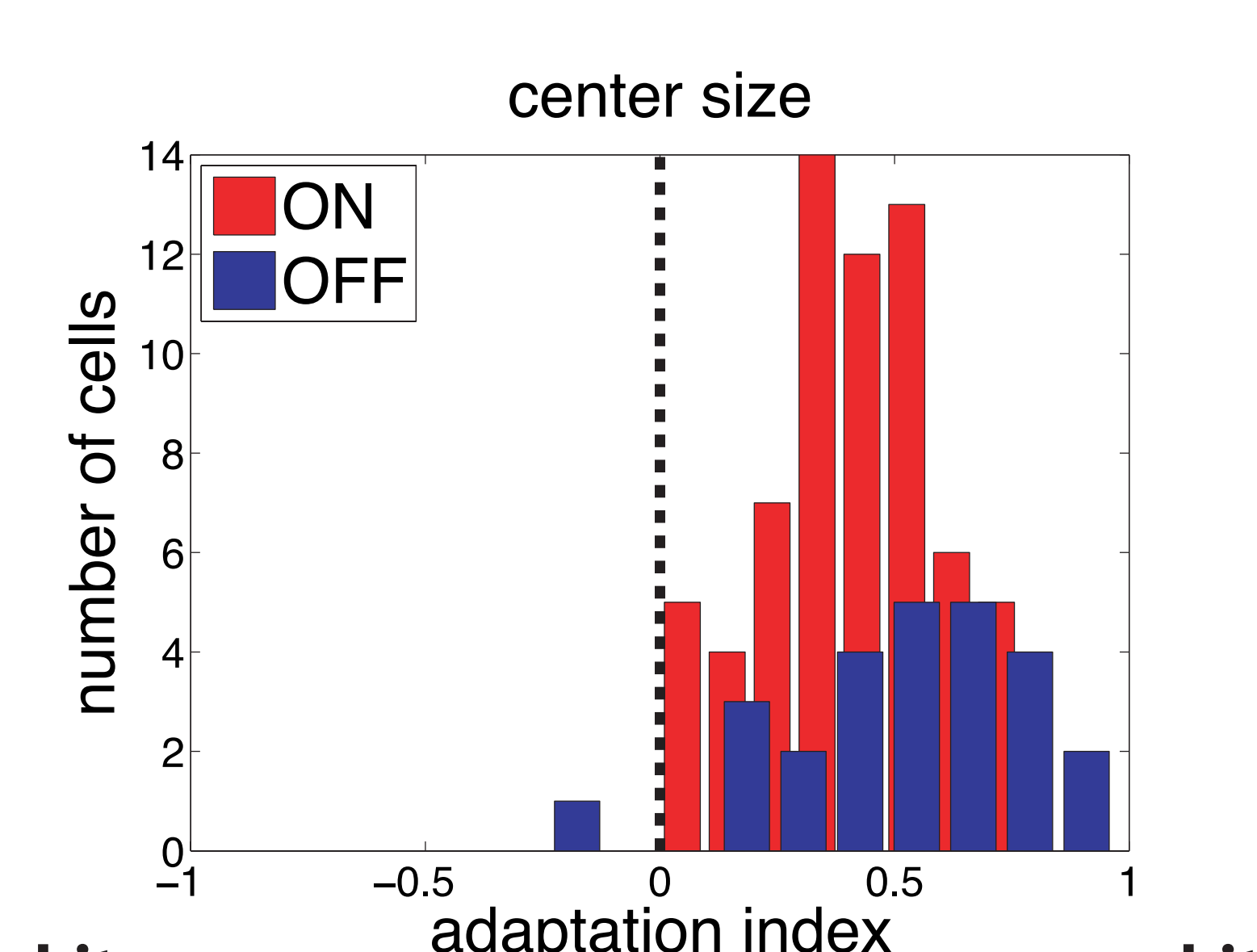
white corr thation ingers

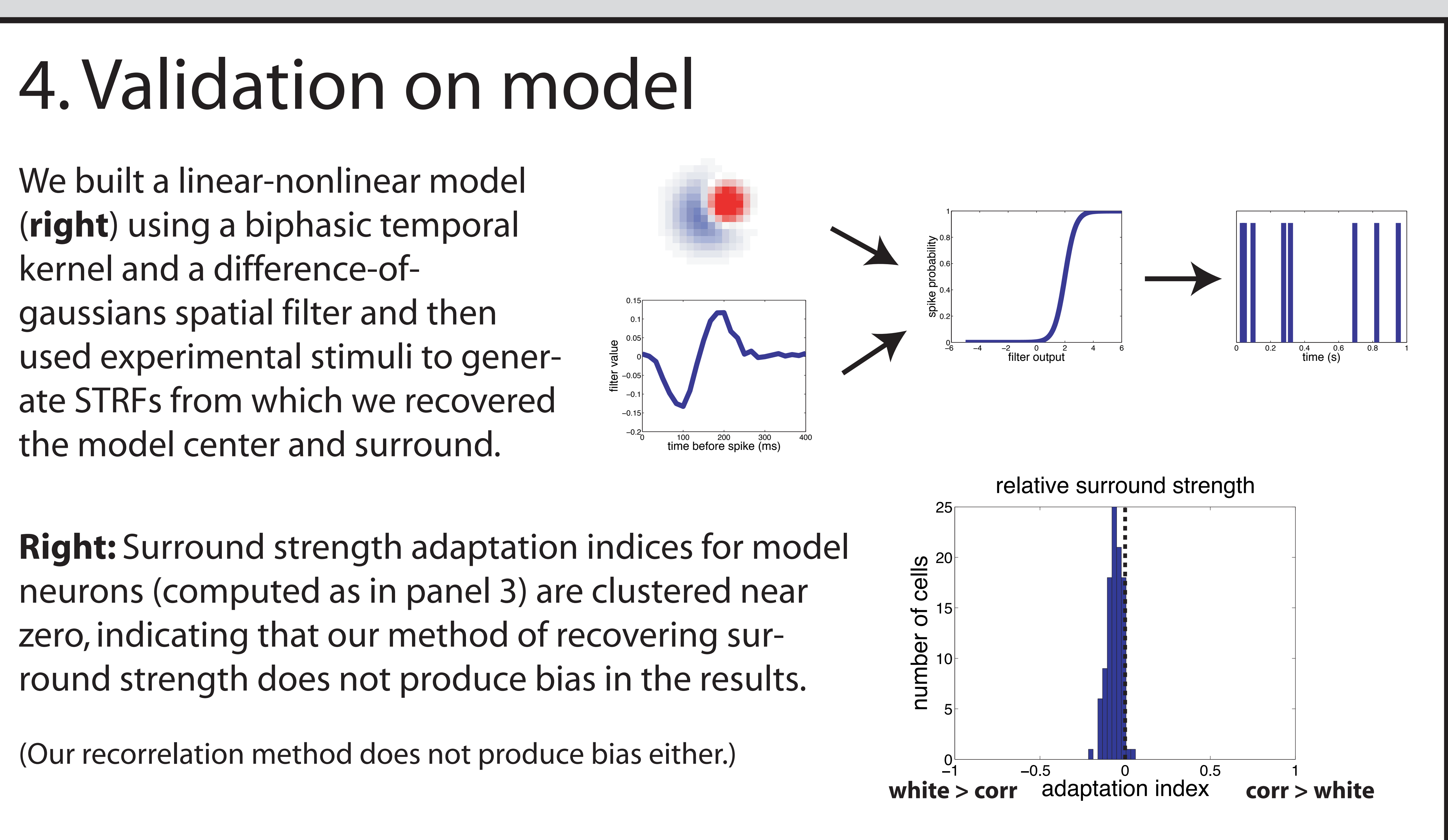

\section{Output correlations}

Pairwise output correlations are similar for exponentially correlated noise and white noise (below middle). Using a more strongly correlated, multiscale stimulus (left), output correltions are stronger than for white noise (right).

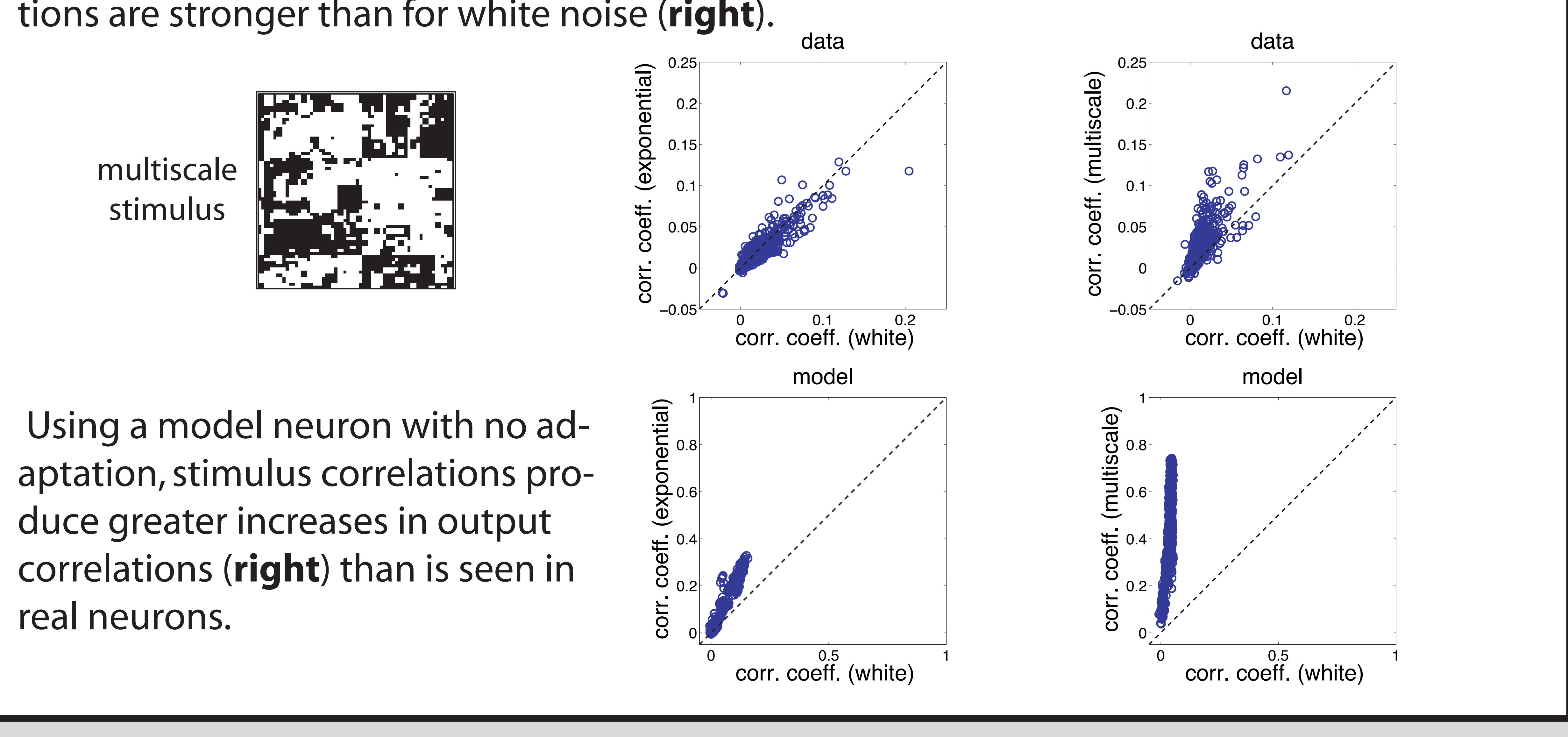

\section{Conclusions}

(A) Relative surround strength is higher during white noise than during correlated noise.

(B) Despite this, retinal output is more decorrelated than in a static linear-nonlinear model. 